\title{
Jamming transition in a two-dimensional traffic flow model
}

\author{
Takashi Nagatani \\ Division of Thermal Science, College of Engineering, Shizuoka University, Hamamatsu 432-8561, Japan
}

(Received 23 November 1998)

\begin{abstract}
Phase transition and critical phenomenon are investigated in the two-dimensional traffic flow numerically and analytically. The one-dimensional lattice hydrodynamic model of traffic is extended to the twodimensional traffic flow in which there are two types of cars (northbound and eastbound cars). It is shown that the phase transition among the freely moving phase, the coexisting phase, and the uniformly congested phase occurs below the critical point. Above the critical point, no phase transition occurs. The value $a_{c}$ of the critical point decreases as increasing fraction $c$ of the eastbound cars for $c \leqslant 0.5$. The linear stability theory is applied. The neutral stability lines are found. The time-dependent Ginzburg-Landau (TDGL) equation is derived by the use of nonlinear analysis. The phase separation lines, the spinodal lines, and the critical point are calculated from the TDGL equation. [S1063-651X(99)00405-5]
\end{abstract}

PACS number(s): 05.90.+m, 05.70.Fh, 05.70.Jk, 89.40.+k

\section{INTRODUCTION}

Recently, traffic problems have attracted considerable attention [1-3]. One-dimensional traffic flow has been investigated by the use of a variety of models [4-27]. The jamming transitions between the freely moving traffic and the jammed traffic have been found in the following onedimensional traffic models: the Nagel-Schreckenberg (cellular automaton) model [7], the car following (dynamic) models [6], and the hydrodynamic model [19]. The transitions have properties very similar to the conventional phase transitions and critical phenomena. In the car following model of the one-dimensional traffic flow, the jamming transition has been described in terms of thermodynamic terminology of phase transitions and critical phenomena [28]. The thermodynamic potential describing the jamming transitions has been found by the analytical method.

On the other hand, two-dimensional traffic flow has been investigated by the use of the cellular automation models [29-35]. Biham, Middelton, and Levine [29] have proposed a two-dimensional traffic cellular automaton model (BML model) for a network of city roads on a square lattice. There are two types of cars in the model: one type of cars (eastbound cars) is able to move only in the positive $x$ direction and the other type of cars (northbound cars) only in the positive $y$ direction. They have found that a phase transition from a freely moving phase to a perfectly jammed phase occurs with increasing car density. The two-dimensional cellular automaton model has been extended to traffic problems with two-level crossing [30], direction changing [31], and car accidents. The cellular automaton models have been analyzed by the use of the mean-field theory $[30,34]$.

One-dimensional traffic flow problems have been investigated extensively by the use of various models, but twodimensional problems have been studied only with use of the cellular automaton model. Until now, the two-dimensional traffic flow problems have seldom been investigated by the hydrodynamic model and the car following model. The hydrodynamic and car following models have the merit that the linear stability analysis and the nonlinear analysis can be applied.
In this paper, we present a lattice hydrodynamic model for two-dimensional traffic flow. We study numerically and analytically the two-dimensional traffic behavior in the lattice hydrodynamic model. We show that the phase transition occurs among the freely moving phase, the coexisting phase, and the uniformly congested phase. We apply the linear stability theory and nonlinear analysis to the lattice hydrodynamic model. We derive the time-dependent GinzburgLandau equation. We calculate the phase separation lines, the spinodal lines, and the critical point. We compare the analytical result with the simulation result.

\section{LATTICE HYDRODYNAMIC MODEL}

We present a lattice hydrodynamic model for twodimensional traffic flow. For simplicity, we consider only two types of cars, similar to the BML model [29]: one type of cars (eastbound cars) moves only in the positive $x$ direction and the other type of cars (northbound cars) moves only in the positive $y$ direction. The continuity equation relates the local density of eastbound cars (northbound cars) to the local average speed. The speed and density of eastbound cars (northbound cars) are denoted, respectively, by $u(x, y, t)$ $[\nu(x, y, t)]$ and $\rho_{x}(x, y, t)\left[\rho_{y}(x, y, t)\right]$. The continuity equations of the eastbound and northbound cars are given, respectively, by

$$
\begin{aligned}
& \partial_{t} \rho_{x}(x, y, t)+\partial_{x} \rho_{x}(x, y, t) u(x, y, t)=0, \\
& \partial_{t} \rho_{y}(x, y, t)+\partial_{y} \rho_{y}(x, y, t) \nu(x, y, t)=0,
\end{aligned}
$$

where $\partial_{t}=\partial / \partial t, \partial_{x}=\partial / \partial x$, and $\partial_{y}=\partial / \partial y$.

We assume that the traffic current is adjusted by the optimal current with a delay time. The traffic currents of eastbound and northbound cars are given, respectively, by

$$
\begin{gathered}
\rho_{x}(x, y, t+\tau) u(x, y, t+\tau)=c \rho_{0} V\left(\rho\left(x+x_{0}, y, t\right)\right), \\
\rho_{y}(x, y, t+\tau) \nu(x, y, t+\tau)=(1-c) \rho_{0} V\left(\rho\left(x, y+y_{0}, t\right)\right),
\end{gathered}
$$


where $c$ is the fraction of eastbound cars, $\rho_{0}$ is the total average density, $\rho(x, y, t)\left[=\rho_{x}(x, y, t)+\rho_{y}(x, y, t)\right]$ is the local density, $x_{0}$ is the average headway of eastbound cars, and $y_{0}$ is the average headway of northbound cars. Equations (3) and (4) are the evolution equations in the place of the Navier-Stokes equation. The function $V(\rho(x, y, t))$ is called the optimal velocity. The delay time $\tau$ allows for the time lag that it takes the traffic current to reach the optimal current when the traffic flow is varying. The idea is that traffic currents $\rho_{x}(x, y, t) u(x, y, t)$ and $\rho_{y}(x, y, t) \nu(x, y, t)$ at position $(x, y)$ at time $t$ are adjusted by the optimal currents $c \rho_{0} V\left(\rho\left(x+x_{0}, y, t-\tau\right)\right)$ at position $\left(x+x_{0}, y\right)$ and (1 -c) $\rho_{0} V\left(\rho\left(x, y+y_{0}, t-\tau\right)\right)$ at position $\left(x, y+y_{0}\right)$ at time $t$ $-\tau$. This is similar to the idea of the one-dimensional car following model analyzed by Newell [4] and Whitham [5]. In the limit of $c=0$ or 1 , the above two-dimensional hydrodynamic model reduces to the one-dimensional hydrodynamic model [36]. In the one-dimensional hydrodynamic model, it has been proved that the jamming transition occurs.

We transform the hydrodynamic model to the lattice model. The time and space derivatives are replaced by the following differences:

$$
\partial_{t} \rho_{x}(x, y, t) \cong \frac{\rho_{x}(x, y, t+\tau)-\rho_{x}(x, y, t)}{\tau},
$$

$$
\begin{aligned}
& \partial_{x} \rho_{x}(x, y, t) u(x, y, t) \\
& \cong \frac{\rho_{x}(x, y, t) u(x, y, t)-\rho_{x}\left(x-x_{0}, y, t\right) u\left(x-x_{0}, y, t\right)}{x_{0}},
\end{aligned}
$$

$\partial_{y} \rho_{y}(x, y, t) \nu(x, y, t)$

$$
\cong \frac{\rho_{y}(x, y, t) \nu(x, y, t)-\rho_{y}\left(x, y-y_{0}, t\right) \nu\left(x, y-y_{0}, t\right)}{y_{0}} .
$$

We choose $1 /\left(c \rho_{0}\right)$ and $1 /\left[(1-c) \rho_{0}\right]$ for $x_{0}$ and $y_{0}$ where $1 /\left(c \rho_{0}\right)$ and $1 /\left[(1-c) \rho_{0}\right]$ are the average headways of the eastbound and northbound cars. We consider the twodimensional traffic flow on the square lattice with the horizontal and vertical spacings: $1 /\left(c \rho_{0}\right)$ and $1 /\left[(1-c) \rho_{0}\right]$. The lattice hydrodynamic model is described by the following difference equations:

$$
\begin{gathered}
\rho_{x, j, m}(t+\tau)-\rho_{x, j, m}(t)+\tau c \rho_{0}\left[\rho_{x, j, m}(t) u_{j, m}(t)\right. \\
\left.-\rho_{x, j-1, m}(t) u_{j-1, m}(t)\right]=0, \\
\rho_{x, j, m}(t+\tau) u_{j, m}(t+\tau)=c \rho_{0} V\left(\rho_{j+1, m}(t)\right)
\end{gathered}
$$

for the eastbound cars, and

$$
\begin{aligned}
& \rho_{y, j, m}(t+\tau)-\rho_{y, j, m}(t)+\tau(1-c) \rho_{0}\left[\rho_{y, j, m}(t) \nu_{j, m}(t)\right. \\
& \left.\quad-\rho_{y, j, m-1}(t) \nu_{j, m-1}(t)\right]=0, \\
& \rho_{y, j, m}(t+\tau) \nu_{j, m}(t+\tau)=(1-c) \rho_{0} V\left(\rho_{j, m+1}(t)\right)
\end{aligned}
$$

for the northbound cars, where $\rho_{x, j, m}(t)$ and $\rho_{y, j, m}(t)$ are the local densities of eastbound and northbound cars on site $(j, m)$ at time $t$.
In the limit of $c=0$ or 1 , the two-dimensional lattice hydrodynamic model reduces to the one-dimensional lattice hydrodynamic model [36].

The optimal velocity function is given by

$$
V\left(\rho_{j, m}(t)\right)=\tanh \left(\frac{2}{\rho_{0}}-\frac{\rho_{j, m}(t)}{\rho_{0}^{2}}-\frac{1}{\rho_{c}}\right)+\tanh \left(\frac{1}{\rho_{c}}\right),
$$

where $\rho_{c}$ is the inverse of the safety distance $[25,28]$. This function has the turning point (inflection point) at $\rho_{j, m}(t)$ $=\rho_{0}=\rho_{c}$. Generally, it is necessary that the optimal velocity function has the following properties: it is a monotonically decreasing function, it has an upper bound (maximal velocity), and it has a turning point at the safety distance.

By inserting Eqs. (9) and (11) into Eqs. (8) and (10), respectively, and adding Eq. (8) to Eq. (10), one obtains the density equation

$$
\begin{gathered}
\rho_{j, m}(t+2 \tau)-\rho_{j, m}(t+\tau)+\tau c^{2} \rho_{0}^{2}\left[V\left(\rho_{j+1, m}(t)\right)\right. \\
\left.-V\left(\rho_{j, m}(t)\right)\right]+\tau(1-c)^{2} \rho_{0}^{2}\left[V\left(\rho_{j, m+1}(t)\right)\right. \\
\left.-V\left(\rho_{j, m}(t)\right)\right]=0,
\end{gathered}
$$

where $\rho_{j, m}(t)=\rho_{x, j, m}(t)+\rho_{y, j, m}(t)$.

In the limit of $c=0$ or 1 , Eq. (13) reduces to the density equation of the one-dimensional traffic flow.

\section{SIMULATION}

We carry out a simulation to study numerically the traffic behaviors in the two-dimensional lattice hydrodynamic model. We derive numerically the phase separation lines (coexisting curves). We compare the simulation result with the analytical result in Sec. V. A priori it cannot be assumed that the lattice hydrodynamic model yields a jamming transition similar to the cellular automata. Therefore, simulation is carried out to validate two points. (1) First it has to be shown that the model is capable of describing two-dimensional traffic dynamics and the jamming transition indeed occurs. (2) Next the applicability of the nonlinear analysis has to be proved.

We set $\tau$ as the unit time step. The boundary is periodic. Initially, the density is assumed to be distributed uniformly over space: $\rho_{j, m}(0)=\rho_{0}=\rho_{c}=0.2$. Then, the local densities $\rho_{L / 2, L / 2}(1)$ and $\rho_{L / 2-1, L / 2-1}(1)$ at sites $(L / 2, L / 2)$ and $(L / 2$ $-1, L / 2-1)$ at time $t=1$ are set as 0.1 and 0.3 where $L$ is the system size. We study the traffic patterns for various values of delay time. As a result, three types of traffic flow have to be distinguished: (1) a freely moving phase, (2) a coexisting phase in which jams appear, and (3) a uniformly congested phase. In Fig. 1, the time evolutions of traffic patterns are shown when the disturbance at the center is added to the uniform initial state $\left(\rho_{0}=0.2\right)$, where the sensitivity $a$ (the inverse of the delay time) is 1.0 and the system size is 140 $\times 140$. The regions with higher density than 0.2 are indicated by the gray color. The gray regions represent the jammed traffic. The patterns (a) and (b) indicate the time evolutions of traffic patterns for $c=0.5$ and 0.2 . At early stage, jams occur within a small region. In time, the jams spread to the whole system. For $c=0.5$ in which the density of eastbound cars equals the density of northbound cars, the jams propa- 

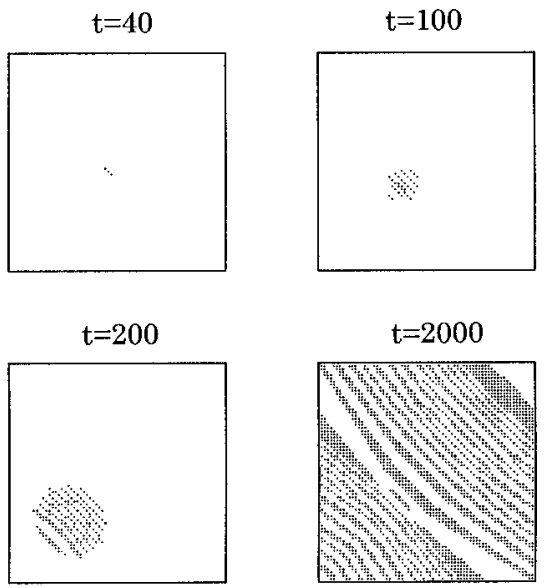

(a)
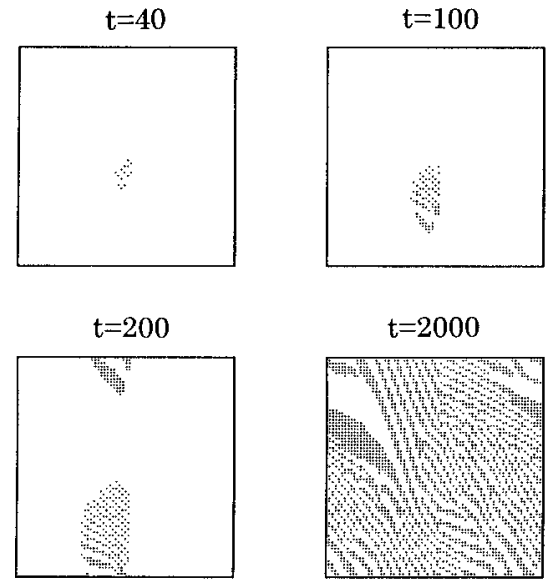

(b)

FIG. 1. Time evolutions of traffic patterns for $t=40,100,200$, and 2000 when the disturbance at the center is added to the uniform initial state $\left(\rho_{0}=0.2\right)$, where the system size is $140 \times 140$. The regions with higher density than $\rho_{0}=0.2$ are indicated by the gray color. The gray regions represent the jammed traffic. (a) The traffic patterns for $a=1.0$ and $c=0.5$. (b) The traffic patterns for $a=1.0$ and $c=0.2$.

gate backward. The traffic jams are formed with the diagonally striped patterns. For $c=0.2$ in which the density of the eastbound cars is less than the density of the northbound cars, the traffic pattern of jams exhibits the complex structure but the jams propagate backward approximately in the diagonal direction. Figure 2 shows the time evolutions of traffic patterns on the single-horizontal road at $y=40$ for the same initial condition as Fig. 1. The patterns (a) and (b) indicate the time evolutions for $c=0.5$ and 0.2 . The jammed regions indicated by the gray propagate backward. Figure 3 shows the density profiles obtained at $t=6000$ on the singlehorizontal road at $y=40$ for the same initial condition as Fig. 2. The profiles (a) and (b) indicate those for $c=0.5$ and 0.2 . The regions of density higher than 0.2 represent the traffic jams and propagate backward. The jams are the density waves. The density waves have the symmetric kink-antikink form after sufficiently long time. After sufficiently long time, the shapes of density waves do not change with time for $c$ $=0.5$ but do change for $c=0.2$.

Figure 4 shows the plots of density difference $\rho(t)-\rho(t$

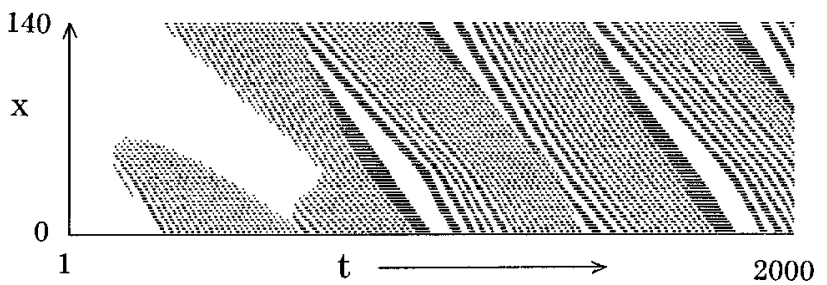

(a)

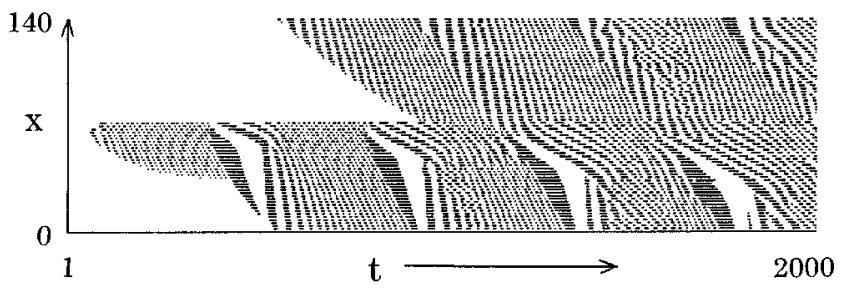

(b)

FIG. 2. The time evolutions of traffic patterns on the horizontal road at $y=40$. The jammed traffic with higher density than $\rho_{0}$ $=0.2$ is indicated by the gray. The jammed regions propagate backward as the density waves. (a) $a=1.0$ and $c=0.5$. (b) $a=1.0$ and $c=0.2$.

-1) versus density $\rho(t)$ at a site of the system for $t$ $=6000-10000$ for $a=1.0$ : (a) $c=0.5$ and (b) $c=0.2$. The plots (a) and (b) correspond, respectively, to the traffic flows (a) and (b) in Fig. 3. The pattern (a) in Fig. 3 exhibits the limit cycle (a single closed curve) in the plot of Fig. 4. It corresponds to the periodic traffic behavior. The pattern (b) in Fig. 3 exhibits the dispersed plots around a closed loop. It corresponds to the irregular traffic behavior. The points on the right and left ends represent, respectively, the states within the traffic jams and within the freely moving phase.
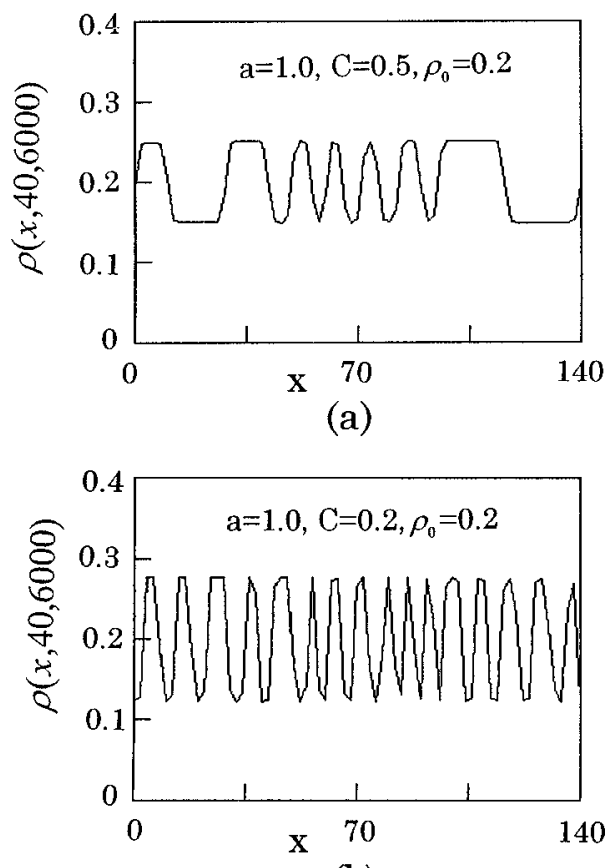

(b)

FIG. 3. The density profiles obtained at $t=6000$ on the horizontal road at $y=40$ for $a=1.0$ and $\rho_{0}=0.2$. The density waves (traffic jams) have the symmetric kink-antikink form. (a) $c=0.5$. (b) $c$ $=0.2$. 


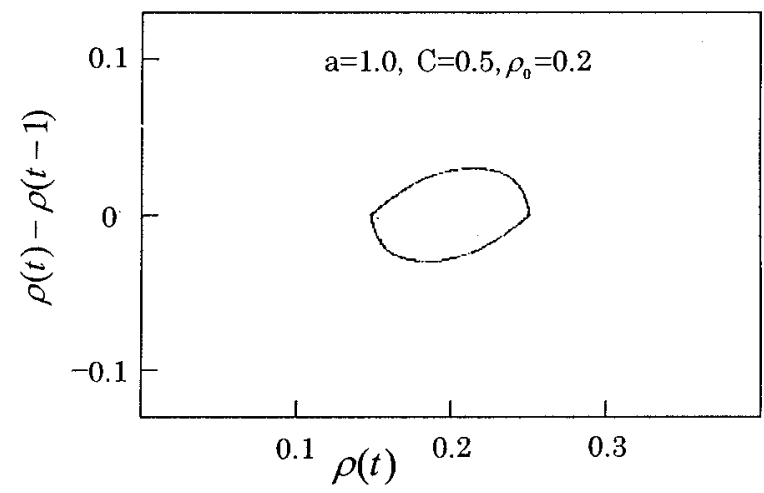

(a)

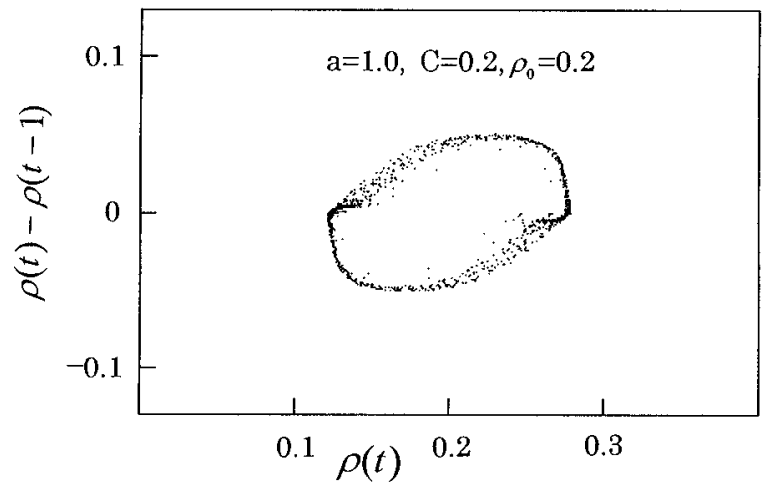

(b)

FIG. 4. The plots of density difference $\rho(t)-\rho(t-1)$ vs density $\rho(t)$ for $t=6000-10000$ where $a=1.0$ and $\rho_{0}=0.2$. (a) $c=0.5$. (b) $c=0.2$.

In any case considering long-time evolution, only two distinct densities survive for the coexisting phase, depending on the sensitivity (the inverse of the delay time) and the fraction $c$. Each density is the density of the transition points on the coexisting curve. Figure 5 shows the plots of densities at the transition points versus sensitivity $a$. The circles, triangles, and diamonds indicate the simulation results, respectively, for $c=0.1,0.2$, and 0.5 . The solid lines represent the analytical results in Sec. V. The simulation results are consistent with the analytical results. The phase separation line decreases as increasing fraction $c$ for $c \leqslant 0.5$. The apex of each curve indicates the critical point. Above the critical point, no traffic jams occur. Figure 6 shows the plot of the critical point versus fraction $c$. The circular points indicate the simulation result. The solid line indicates the analytical result in Sec. V. The simulation result agrees with the analytical result.

\section{LINEAR STABILITY ANALYSIS}

We apply the linear stability method to Eq. (13). We consider the stability of the uniform traffic flow. The uniform traffic flow is defined by such a state as a traffic flow with constant density $\rho_{0}$, constant $x$-directional velocity $V\left(\rho_{0}\right)$, and constant $y$-directional velocity $V\left(\rho_{0}\right)$. Equation (13) has the solution of the uniform steady state:

$$
\rho_{j, m}(t)=\rho_{0}, \quad u_{j, m}(t)=V\left(\rho_{0}\right), \text { and } \quad \nu_{j, m}(t)=V\left(\rho_{0}\right)
$$

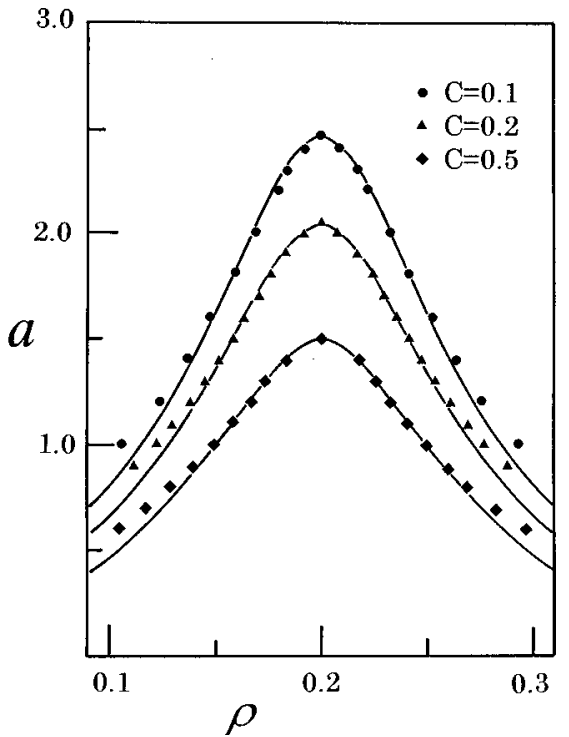

FIG. 5. Phase separation lines in the $(\rho, a)$ plane, where $\rho$ is the density, $a(=1 / \tau)$ is the sensitivity (the inverse of the delay time), and $\rho_{c}=0.2$. The circles, triangles, and diamonds indicate, respectively, the simulation results for $c=0.1,0.2$, and 0.5 . The solid lines represent the analytical results obtained by the nonlinear analysis.

Let $y_{j, m}(t)$ be a small deviation from the uniform steadystate flow: $\rho_{j, m}(t)=\rho_{0}+y_{j, m}(t)$. Then, the linear equation is obtained from Eq. (13),

$$
\begin{aligned}
y_{j, m}( & t+2 \tau)-y_{j, m}(t+\tau)+\tau c^{2} \rho_{0}^{2} V^{\prime}\left(\rho_{0}\right) \\
& \times\left[y_{j+1, m}(t)-y_{j, m}(t)\right] \\
& +\tau(1-c)^{2} \rho_{0}^{2} V^{\prime}\left(\rho_{0}\right)\left[y_{j, m+1}(t)-y_{j, m}(t)\right]=0,
\end{aligned}
$$

where $V^{\prime}\left(\rho_{0}\right)=\left.[d V(\rho) / d \rho]\right|_{\rho=\rho_{0}}$

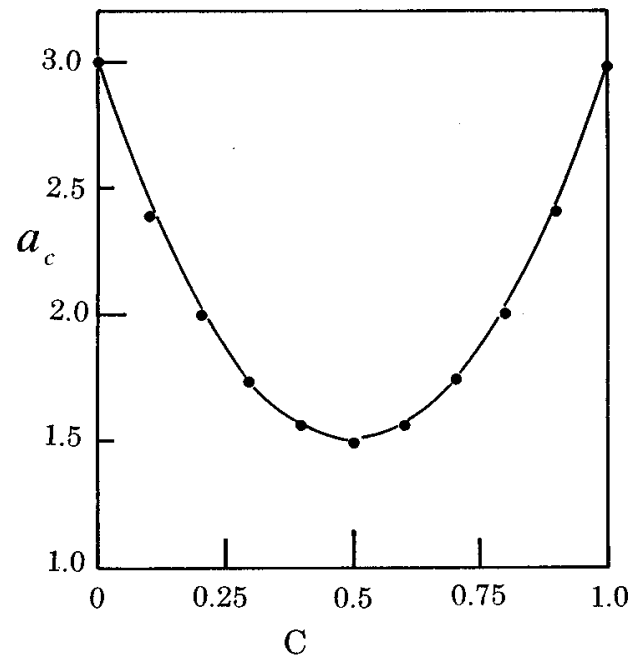

FIG. 6. The plot of the critical points vs fraction $c$. The circles indicate the simulation result. The solid line represents the analytical result obtained by the nonlinear analysis. 


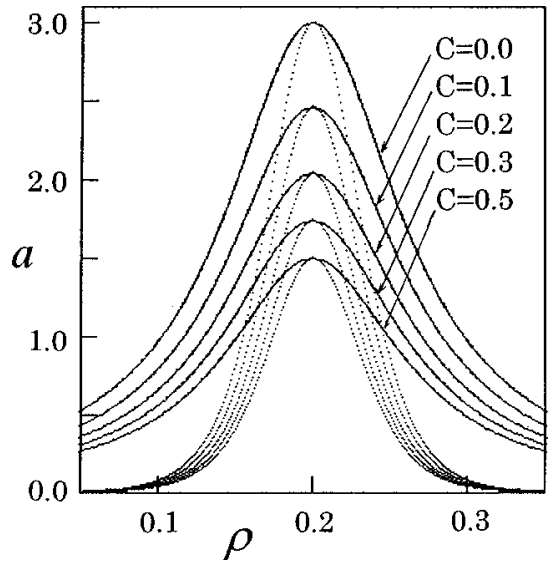

FIG. 7. The phase diagrams in the $(\rho, a)$ plane obtained by the TDGL equation for $c=0.0,0.1,0.2,0.3$, and 0.5 . The solid and dotted lines indicate the phase separation lines and the spinodal curves. The apex of each curve indicates the critical point.

Since the density wave propagates in both negative $x$ and $y$ directions with equivalent propagation velocity, we expand $y_{j, m}(t)$ as follows: $y_{j, m}(t) \propto \exp \{i k(j+m)+z t\}$. The following equation of $z$ is derived:

$$
e^{2 z \tau}-e^{z \tau}+\tau\left\{c^{2}+(1-c)^{2}\right\} \rho_{0}^{2} V^{\prime}\left(e^{i k}-1\right)=0,
$$

where $V^{\prime}=V^{\prime}\left(\rho_{0}\right)$.

By expanding $z$ with $z_{1}(i k)+z_{2}(i k)^{2}+\cdots$, the first-order and second-order terms of $i k$ are obtained,

$$
\begin{gathered}
z_{1}=-\left\{c^{2}+(1-c)^{2}\right\} \rho_{0}^{2} V^{\prime}, \\
z_{2}=-\frac{3}{2} \tau\left\{c^{2}+(1-c)^{2}\right\}^{2}\left(\rho_{0}^{2} V^{\prime}\right)^{2}-\frac{\left\{c^{2}+(1-c)^{2}\right\}}{2} \rho_{0}^{2} V^{\prime} .
\end{gathered}
$$

If $z_{2}$ is a negative value, the uniform steady-state flow becomes unstable for long-wavelength modes. When $z_{2}$ is a positive value, the uniform flow is stable. One obtains the neutral stability condition

$$
\tau=-\frac{1}{3\left\{c^{2}+(1-c)^{2}\right\} \rho_{0}^{2} V^{\prime}} .
$$

For small disturbances of long wavelengths, the uniform traffic flow is unstable if

$$
\tau>-\frac{1}{3\left\{c^{2}+(1-c)^{2}\right\} \rho_{0}^{2} V^{\prime}} .
$$

The derivative $V^{\prime}$ of optimal velocity has the minimal value at turning point $\rho_{0}=\rho_{c}$. If $\tau<\tau_{c}\left[\tau_{c}=-1 / 3\left\{c^{2}+(1\right.\right.$ $\left.\left.-c)^{2}\right\} \rho_{0}^{2} V^{\prime}\left(\rho_{c}\right)=1 / 3\left\{c^{2}+(1-c)^{2}\right\}\right]$, the uniform flow is always stable irrespective of density. We find that there is a critical point at $\rho=\rho_{c}$ and $\tau=\tau_{c}$. When $c=0$ or 1, the critical point and the neutral stability line are consistent with those in the one-dimensional traffic model [36]. The dotted lines in Fig. 7 show the neutral stability lines for $c=0.0,0.1$, $0.2,0.3$, and 0.5 . The apex of each curve indicates the critical point. Above each curve, the two-dimensional traffic flow is stable and the density wave (traffic jam) does not appear. Below each curve, the traffic flow is unstable and the density wave appears. As fraction $c$ increases $(c<0.5)$, the critical points and the neutral stability curves decrease.

\section{TDGL EQUATION}

We now consider the traffic behavior of long-wavelength modes on coarse-grained scales. The simplest way to describe the behavior of long-wavelength modes is the longwave expansion. We consider the slowly varying behavior at long wavelengths near the critical point $\left(\rho_{c}, \tau_{c}\right)$. We extract slow scales for space variables $j, m$, and time variable $t$ $[28,37]$. For $0<\varepsilon \ll 1$, we therefore define the slow variables $X$ and $T$ :

$$
X=\varepsilon(j+m+b t) \text { and } T=\varepsilon^{3} t,
$$

where $b$ is a constant determined later. Here we consider the density wave propagating in the diagonal direction. The diagonally propagating density wave is observed in our simulation. We set the density as

$$
\rho_{j, m}(t)=\rho_{c}+\varepsilon R(X, T) .
$$

By expanding Eq. (13) to the fifth order of $\varepsilon$ with the use of Eqs. (20) and (21), one obtains the following nonlinear partial differential equations:

$$
\begin{aligned}
\varepsilon^{2}\left(b+g \rho_{c}^{2} V^{\prime}\right) \partial_{X} R+\varepsilon^{3}\left(\frac{3 b^{2} \tau}{2}+\frac{g \rho_{c}^{2} V^{\prime}}{2}\right) \partial_{X}^{2} R \\
+\varepsilon^{4}\left[\partial_{T} R+\left(\frac{7 b^{3} \tau^{2}}{6}+\frac{g \rho_{c}^{2} V^{\prime}}{6}\right) \partial_{X}^{3} R+\frac{g \rho_{c}^{2} V^{\prime \prime \prime}}{6} \partial_{X} R^{3}\right] \\
+\varepsilon^{5}\left[3 b \tau \partial_{T} \partial_{X} R+\left(\frac{5 b^{4} \tau^{3}}{8}+\frac{g \rho_{c}^{2} V^{\prime}}{24}\right) \partial_{X}^{4} R\right. \\
\left.+\frac{g \rho_{c}^{2} V^{\prime \prime \prime}}{12} \partial_{X}^{2} R^{3}\right]=0,
\end{aligned}
$$

where $V^{\prime}=\left.[d V(\rho) / d \rho]\right|_{\rho=\rho_{c}}, V^{\prime \prime \prime}=\left.\left[d^{3} V(\rho) / d \rho^{3}\right]\right|_{\rho=\rho_{c}}$, and $g=c^{2}+(1-c)^{2}$. Here we used the expansions shown in the Appendix.

By taking $b=-g \rho_{c}^{2} V^{\prime}\left(\rho_{c}\right)$, the second-order term of $\varepsilon$ is eliminated from Eq. (22). We consider the neighborhood of the critical point $\tau_{c}$ :

$$
\frac{\tau}{\tau_{c}}=1+\varepsilon^{2},
$$

where $\tau_{c}=-1 / 3 g \rho_{c}^{2} V^{\prime}\left(\rho_{c}\right)=1 / 3 g$. Equation (22) is rewritten

$$
\begin{aligned}
\varepsilon^{4} \partial_{T} R= & \varepsilon^{4} \frac{g\left(-\rho_{c}^{2} V^{\prime}\right)}{27} \partial_{X}^{3} R-\varepsilon^{4} \frac{g \rho_{c}^{2} V^{\prime \prime \prime}}{6} \partial_{X} R^{3} \\
& -\varepsilon^{3} \frac{g\left(-\rho_{c}^{2} V^{\prime}\right)}{2}\left(\frac{\tau}{\tau_{c}}-1\right) \partial_{X}^{2} R-\varepsilon^{5} \frac{g\left(-\rho_{c}^{2} V^{\prime}\right)}{54} \partial_{X}^{4} R \\
& +\varepsilon^{5} \frac{g \rho_{c}^{2} V^{\prime \prime \prime}}{12} \partial_{X}^{2} R^{3} .
\end{aligned}
$$


By transforming variables $X$ and $T$ to variables $x=\varepsilon^{-1} X$ and $t=\varepsilon^{-3} T$, and taking $S(x, t)=\varepsilon R(X, T)$, Eq. (24) is rewritten as follows:

$$
\begin{aligned}
\partial_{t} S= & \frac{g\left(-\rho_{c}^{2} V^{\prime}\right)}{27} \partial_{x}^{3} S-\frac{g \rho_{c}^{2} V^{\prime \prime \prime}}{6} \partial_{x} S^{3} \\
& -\frac{g\left(-\rho_{c}^{2} V^{\prime}\right)}{2}\left(\frac{\tau}{\tau_{c}}-1\right) \partial_{x}^{2} S-\frac{g\left(-\rho_{c}^{2} V^{\prime}\right)}{54} \partial_{x}^{4} S \\
& +\frac{g \rho_{c}^{2} V^{\prime \prime \prime}}{12} \partial_{x}^{2} S^{3} .
\end{aligned}
$$

By adding term $g\left(-\rho_{c}^{2} V^{\prime}\right)\left(\tau / \tau_{c}-1\right) \partial_{x} S$ on both left and right sides of Eq. (25) and performing the transformation $t_{1}$ $=t$ and $x_{1}=x+g\left(-\rho_{c}^{2} V^{\prime}\right)\left(\tau / \tau_{c}-1\right) t$ in Eq. (25), one obtains

$$
\begin{aligned}
\partial_{t_{1}} S= & \left(\partial_{x_{1}}-\frac{1}{2} \partial_{x_{1}}^{2}\right)\left[\frac{g\left(-\rho_{c}^{2} V^{\prime}\right)}{27} \partial_{x_{1}}^{2} S\right. \\
& \left.+g\left(-\rho_{c}^{2} V^{\prime}\right)\left(\frac{\tau}{\tau_{c}}-1\right) S-\frac{g \rho_{c}^{2} V^{\prime \prime \prime}}{6} S^{3}\right] .
\end{aligned}
$$

We define the thermodynamic potential

$$
\phi(S) \equiv-\frac{g\left(-\rho_{c}^{2} V^{\prime}\right)}{2}\left(\frac{\tau}{\tau_{c}}-1\right) S^{2}+\frac{g \rho_{c}^{2} V^{\prime \prime \prime}}{24} S^{4} .
$$

By rewriting Eq. (26) with Eq. (27), one obtains the timedependent Ginzburg-Landau (TDGL) equation:

$$
\begin{gathered}
\partial_{t_{1}} S=-\left(\partial_{x_{1}}-\frac{1}{2} \partial_{x_{1}}^{2}\right) \frac{\delta \Phi(S)}{\delta S}, \\
\text { with } \Phi(S) \equiv \int d x_{1}\left(\frac{g\left(-\rho_{c}^{2} V^{\prime}\right)}{54}\left(\partial_{x_{1}} S\right)^{2}+\phi(S)\right),
\end{gathered}
$$

where $\phi(S)$ is given by Eq. (27).

The TDGL equation (28) has two steady-state solutions in addition to a trivial solution $S=0$ : the one is the uniform solution

$$
S\left(x_{1}, t_{1}\right)= \pm\left[\frac{6\left(-\rho_{c}^{2} V^{\prime}\right)\left(-3 g \rho_{c}^{2} V^{\prime} \tau-1\right)}{\rho_{c}^{2} V^{\prime \prime \prime}}\right]^{1 / 2}
$$

and the other is the kink solution

$$
\begin{aligned}
S\left(x_{1}, t_{1}\right)= & \pm\left[\frac{6\left(-\rho_{c}^{2} V^{\prime}\right)\left(-3 g \rho_{c}^{2} V^{\prime} \tau-1\right)}{\rho_{c}^{2} V^{\prime \prime \prime}}\right]^{1 / 2} \\
& \times \tanh \left[\left(\frac{27}{2}\left(-3 g \rho_{c}^{2} V^{\prime} \tau-1\right)\right)^{1 / 2}\left(x_{1}-x_{1_{0}}\right)\right],
\end{aligned}
$$

where $x_{1_{0}}$ is a constant. Equation (30) represents the coexisting phase which consists of a low density phase (freely moving phase) and a high density phase (congested phase).
One can obtain the coexisting curve, the spinodal line, and the critical point by differentiating thermodynamic potential (27) with order parameter $S$. The coexisting curve is given by the condition

$$
\frac{\partial \phi}{\partial S}=0 \text { and } \frac{\partial^{2} \phi}{\partial S^{2}}>0 .
$$

From Eq. (31), one obtains the coexisting curve in terms of the original parameters

$$
\rho_{\mathrm{co}}=\rho_{c} \pm\left[\frac{6\left(-\rho_{c}^{2} V^{\prime}\right)\left(-3 g \rho_{c}^{2} V^{\prime} \tau-1\right)}{\rho_{c}^{2} V^{\prime \prime \prime}}\right]^{1 / 2} .
$$

The spinodal line is given by the condition

$$
\frac{\partial^{2} \phi}{\partial S^{2}}=0
$$

From Eq. (33), one obtains the spinodal line in terms of the original parameters

$$
\rho_{\mathrm{sp}}=\rho_{c} \pm\left[\frac{2\left(-\rho_{c}^{2} V^{\prime}\right)\left(-3 g \rho_{c}^{2} V^{\prime} \tau-1\right)}{\rho_{c}^{2} V^{\prime \prime \prime}}\right]^{1 / 2} .
$$

The critical point is given by the condition

$$
\frac{\partial \phi}{\partial S}=0 \text { and } \frac{\partial^{2} \phi}{\partial S^{2}}=0 .
$$

From Eq. (35), one obtains the critical point in terms of the original parameters

$$
\rho=\rho_{c} \text { and } \tau_{c}=\frac{1}{3 g\left(-\rho_{c}^{2} V^{\prime}\right)} .
$$

Figure 7 shows the phase diagram in the $(\rho, a)$ plane where $\rho_{c}=0.2,-\rho_{c}^{2} V^{\prime}=1$, and $\rho_{c}^{6} V^{\prime \prime \prime}=2$. Each solid curve indicates the coexisting curve given by Eq. (32). Each dotted line indicates the spinodal line given by Eq. (34). The apex of each curve indicates the critical point. In the region within the coexisting curve, the freely moving phase coexists with the congested phase. The intermediate regions between the coexisting curve and the spinodal line represent the metastable regions.

Generally, the jamming transition is the first-order phase transition below the critical point. The metastability observed in the traffic flow model corresponds to the spinodal decomposition in the conventional first-order phase transition.

\section{MKdV EQUATION}

We derive the modified Korteweg-de Vries equation $(\mathrm{MKdV})$ equation from Eq. (13). We show the connection between the MKdV equation and the TDGL equation. Similarly to the derivation of the TDGL equation, we consider the slowly varying behavior at long wavelengths near the critical point. We extract slow scales for space variables $j, m$, and time $t$. We obtain Eq. (22). By inserting $-3 g \rho_{c}^{2} V^{\prime} \tau-1$ $=\tau / \tau_{c}-1 \equiv \varepsilon^{2}$ into Eq. (23), one obtains 


$$
\begin{aligned}
\varepsilon^{4} \partial_{T} R= & \varepsilon^{4} \frac{g\left(-\rho_{c}^{2} V^{\prime}\right)}{27} \partial_{X}^{3} R-\varepsilon^{4} \frac{g \rho_{c}^{2} V^{\prime \prime \prime}}{6} \partial_{X} R^{3} \\
& -\varepsilon^{5} \frac{g\left(-\rho_{c}^{2} V^{\prime}\right)}{2} \partial_{X}^{2} R-\varepsilon^{5} \frac{g\left(-\rho_{c}^{2} V^{\prime}\right)}{54} \partial_{X}^{4} R \\
& +\varepsilon \frac{g \rho_{c}^{2} V^{\prime \prime \prime}}{12} \partial_{X}^{2} R^{3} .
\end{aligned}
$$

In order to derive the regularized equation, we make the following transformations:

$$
T^{\prime}=\frac{g\left(-\rho_{c}^{2} V^{\prime}\right)}{27} T \text { and } R(X, T)=\left(\frac{-2 \rho_{c}^{2} V^{\prime}}{9 \rho_{c}^{2} V^{\prime \prime \prime}}\right)^{1 / 2} R^{\prime}\left(X, T^{\prime}\right)
$$

One obtains the regularized equation

$$
\partial_{T^{\prime}} R^{\prime}=\partial_{X}^{3} R^{\prime}-\partial_{X} R^{\prime 3}-\varepsilon\left[\frac{27}{2} \partial_{X}^{2} R^{\prime}+\frac{1}{2} \partial_{X}^{4} R^{\prime}-\frac{1}{2} \partial_{X}^{2} R^{\prime 3}\right] .
$$

If we ignore the $O(\varepsilon)$ term in Eq. (39), it is just the MKdV equation with a kink solution as the desired solution,

$$
R_{0}^{\prime}\left(X, T^{\prime}\right)=\sqrt{p} \tanh \sqrt{p / 2}\left(X-p T^{\prime}\right) .
$$

Next, assuming that $R^{\prime}\left(X, T^{\prime}\right)=R_{0}^{\prime}\left(X, T^{\prime}\right)+\varepsilon R_{1}^{\prime}\left(X, T^{\prime}\right)$, we take into account the $O(\varepsilon)$ correction. In order to determine the selected value of the propagation velocity $p$ for the kink solution (40), it is necessary to satisfy the solvability condition

$$
\left(R_{0}^{\prime}, M\left[R_{0}^{\prime}\right]\right) \equiv \int_{-\infty}^{+\infty} d X R_{0}^{\prime} M\left[R_{0}^{\prime}\right]=0,
$$

where $M\left[R_{0}^{\prime}\right]=\frac{27}{2} \partial_{X}^{2} R^{\prime}+\frac{1}{2} \partial_{X}^{4} R^{\prime}-\frac{1}{2} \partial_{X}^{2} R^{\prime 3}$. By performing the integration, we obtain the selected velocity $p=27$. One obtains the solution

$$
R(X, T)=\left(\frac{6\left(-\rho_{c}^{2} V^{\prime}\right)}{\rho_{c}^{2} V^{\prime \prime \prime}}\right)^{1 / 2} \tanh \left(\frac{27}{2}\right)^{1 / 2}\left[X-g\left(-\rho_{c}^{2} V^{\prime}\right) T\right] .
$$

By replacing variables $X$ and $T$ with original variables $j, m$, and $t$, one obtains the solution

$$
\begin{aligned}
\rho_{j, m}(t)= & \rho_{c} \pm\left[\frac{6\left(-\rho_{c}^{2} V^{\prime}\right)\left(-3 g \rho_{c}^{2} V^{\prime} \tau-1\right)}{\rho_{c}^{2} V^{\prime \prime \prime}}\right]^{1 / 2} \\
& \times \tanh \left[\{ \frac { 2 7 } { 2 } ( - 3 g \rho _ { c } ^ { 2 } V ^ { \prime } \tau - 1 ) \} ^ { 1 / 2 } \left\{j+m-g \rho_{c}^{2} V^{\prime}\right.\right. \\
& \left.\left.\times\left(2+3 g \rho_{c}^{2} V^{\prime} \tau\right) t\right\}\right] .
\end{aligned}
$$

The solution (43) agrees with the solution (30) obtained from the TDGL equation. The kink solution represents the coexisting phase which consists of the freely moving phase (with low density) and the congested phase (with high density). The traffic jam seems to be static from the point of view of the backward moving frame with the jam propagation velocity. Thus, the jamming transition can be described by both the TDGL equation with a nontraveling solution and the MKdV equation with a propagating solution.

\section{SUMMARY}

We have proposed a lattice hydrodynamic model for twodimensional traffic flow. We have investigated the jamming transition between the freely moving phase and the jammed phase numerically and analytically. In the numerical simulation, we have shown that the jamming transition occurs with increasing density. We have found that there is a critical point. The critical point decreases as increasing fraction of the eastbound cars.

We have applied the linear stability theory and nonlinear analysis to two-dimensional traffic flow. We have found that the jamming transition is described by the time-dependent Ginzburg-Landau equation. The phase separation lines, the spinodal lines, and the critical point are calculated by the use of the thermodynamic potential. The critical point, the spinodal lines, and the phase separation lines are definitely different from those of one-dimensional traffic flow.

\section{APPENDIX}

In this appendix, we present the expansions of each term in Eq. (13) to fifth order of $\varepsilon$,

$$
\begin{aligned}
\rho_{j, m}(t+\tau)= & \rho_{c}+\varepsilon R+\varepsilon^{2} b \tau \partial_{X} R+\varepsilon^{3} \frac{(b \tau)^{2}}{2} \partial_{X}^{2} R \\
& +\varepsilon^{4} \frac{(b \tau)^{3}}{6} \partial_{X}^{3} R+\varepsilon^{4} \tau \partial_{T} R+\varepsilon^{5} \frac{(b \tau)^{4}}{24} \partial_{X}^{4} R \\
& +\varepsilon^{5} b \tau^{2} \partial_{T} \partial_{X} R,
\end{aligned}
$$

$$
\begin{aligned}
\rho_{j, m}(t+2 \tau)= & \rho_{c}+\varepsilon R+\varepsilon^{2} 2 b \tau \partial_{X} R+\varepsilon^{3} \frac{(2 b \tau)^{2}}{2} \partial_{X}^{2} R \\
& +\varepsilon^{4} \frac{(2 b \tau)^{3}}{6} \partial_{X}^{3} R+\varepsilon^{4} 2 \tau \partial_{T} R+\varepsilon^{5} \frac{(2 b \tau)^{4}}{24} \partial_{X}^{4} R \\
& +\varepsilon^{5} 4 b \tau^{2} \partial_{T} \partial_{X} R,
\end{aligned}
$$

$$
\rho_{j+1, m}(t)=\rho_{c}+\varepsilon R+\varepsilon^{2} \partial_{X} R+\frac{\varepsilon^{3}}{2} \partial_{X}^{2} R+\frac{\varepsilon^{4}}{6} \partial_{X}^{3} R+\frac{\varepsilon^{5}}{24} \partial_{X}^{4} R .
$$

We expand the optimal velocity function at the turning point:

$$
V\left(\rho_{j, m}\right)=V\left(\rho_{c}\right)+V^{\prime}\left(\rho_{c}\right)\left(\rho_{j, m}-\rho_{c}\right)+\frac{V^{\prime \prime \prime}\left(\rho_{c}\right)}{6}\left(\rho_{j, m}-\rho_{c}\right)^{3},
$$




$$
\begin{aligned}
V\left(\rho_{j+1, m}\right)= & V\left(\rho_{c}\right)+V^{\prime}\left(\rho_{c}\right)\left(\rho_{j+1, m}-\rho_{c}\right)+\frac{V^{\prime \prime \prime}\left(\rho_{c}\right)}{6} \\
& \times\left(\rho_{j+1, m}-\rho_{c}\right)^{3} \\
V\left(\rho_{j+1, m}\right)-V\left(\rho_{j, m}\right)= & V^{\prime}\left(\rho_{c}\right)\left(\varepsilon^{2} \partial_{X} R+\frac{\varepsilon^{3}}{2} \partial_{X}^{2} R\right. \\
& \left.+\frac{\varepsilon^{4}}{6} \partial_{X}^{3} R+\frac{\varepsilon^{5}}{24} \partial_{X}^{4} R\right) \\
& +\frac{V^{\prime \prime \prime}\left(\rho_{c}\right)}{6}\left[\varepsilon^{4} \partial_{X} R^{3}+\varepsilon^{5} \frac{1}{2} \partial_{X}^{2} R^{3}\right] .
\end{aligned}
$$

$$
\begin{aligned}
V\left(\rho_{j, m+1}\right)-V\left(\rho_{j, m}\right)= & V^{\prime}\left(\rho_{c}\right)\left(\varepsilon^{2} \partial_{X} R+\frac{\varepsilon^{3}}{2} \partial_{X}^{2} R\right. \\
& \left.+\frac{\varepsilon^{4}}{6} \partial_{X}^{3} R+\frac{\varepsilon^{5}}{24} \partial_{X}^{4} R\right) \\
& +\frac{V^{\prime \prime \prime}\left(\rho_{c}\right)}{6}\left[\varepsilon^{4} \partial_{X} R^{3}+\varepsilon^{5} \frac{1}{2} \partial_{X}^{2} R^{3}\right] .
\end{aligned}
$$

By inserting Eqs. (A1)-(A7) into Eq. (13), one obtains Eq. (22).
[1] Traffic and Granular Flow, edited by D. E. Wolf, M. Schreckenberg, and A. Bachem (World Scientific, Singapore, 1996).

[2] D. Helbing, Verkehrsdynamik (Springer, Berlin, 1997).

[3] Traffic and Granular Flow, edited by M. Schreckenberg and D. E. Wolf (Springer, Singapore, 1998).

[4] G. F. Newell, Oper. Res. 9, 209 (1961).

[5] G. B. Whitham, Proc. R. Soc. London, Ser. A 428, 49 (1990).

[6] M. Bando, K. Hasebe, A. Nakayama, A. Shibata, and Y. Sugiyama, Phys. Rev. E 51, 1035 (1995).

[7] K. Nagel and M. Schreckenberg, J. Phys. I 2, 2221 (1992).

[8] M. Schreckenberg, A. Schadschneider, K. Nagel, and N. Ito, Phys. Rev. E 51, 2939 (1995).

[9] G. Csanyi and J. Kertestz, J. Phys. A 28, 427 (1995).

[10] S. C. Benjamin, N. F. Johnson, and P. M. Hui, J. Phys. A 29, 3119 (1996).

[11] A. Schadschneider and M. Schreckenberg, Ann. Phys. (N.Y.) 6, 541 (1997).

[12] E. Ben-Naim, P. L. Krapivsky, and S. Redner, Phys. Rev. E 50, 822 (1994).

[13] T. Nagatani, Phys. Rev. E 51, 922 (1995).

[14] I. Prigogine and R. Herman, Kinetic Theory of Vehicular Traffic (Elsevier, New York, 1971).

[15] S. L. Paveri-Fontana, Transp. Res. 9, 225 (1975).

[16] D. Helbing, Phys. Rev. E 53, 2366 (1996).

[17] D. Helbing, Physica A 233, 253 (1996).

[18] T. Nagatani, Physica A 237, 67 (1997).
[19] B. S. Kerner and P. Konhauser, Phys. Rev. E 48, 2335 (1993).

[20] B. S. Kerner, P. Konhauser, and M. Schilke, Phys. Rev. E 51, 6243 (1995).

[21] S. Krauss, P. Wagner, and C. Gawron, Phys. Rev. E 55, 5597 (1997).

[22] B. S. Kerner and H. Rehborn, Phys. Rev. E 53, R1297 (1996).

[23] D. A. Kurtze and D. C. Hong, Phys. Rev. E 52, 218 (1995).

[24] T. Komatsu and S. Sasa, Phys. Rev. E 52, 5574 (1995).

[25] T. Nagatani and K. Nakanishi, Phys. Rev. E 57, 6415 (1998).

[26] A. Awazu, J. Phys. Soc. Jpn. 67, 1071 (1998).

[27] K. Nagel, D. E. Wolf, P. Wagner, and P. Simon, Phys. Rev. E 58, 1425 (1998).

[28] T. Nagatani, Phys. Rev. E 58, 4271 (1998).

[29] O. Biham, A. A. Middelton, and D. A. Levine, Phys. Rev. A 46, R6124 (1992).

[30] T. Nagatani, Phys. Rev. E 48, 3290 (1993).

[31] J. A. Cuesta, F. C. Martinez, J. M. Molera, and A. Sanchez, Phys. Rev. E 48, 4175 (1993).

[32] S. Tadaki and M. Kikuchi, Phys. Rev. E 50, 4564 (1994).

[33] J. M. Molera, F. C. Martinez, J. A. Cuesta, and R. Brito, Phys. Rev. E 51, 175 (1995).

[34] K. H. Chung, P. M. Hui, and G. Q. Gu, Phys. Rev. E 51, 772 (1995).

[35] T. Horiguchi and T. Sakakibara, Physica A 252, 388 (1998).

[36] T. Nagatani, Physica A 264, 581 (1999).

[37] M. C. Cross and P. C. Hohenberg, Rev. Mod. Phys. 65, 851 (1993). 